Geometrical Mechanics and de Broglie Waves

By Prof. J. L. Synge. (Cambridge Monographs on Mechanics and Applied Mathematies.) Pp. vii + 167. (Cambridge: At the University Press, 1954.) 25s. net.

CEOMETRICAL optics as well as Newtonian T mechanics are based on the same variational principle $\delta \int v \mathrm{~d} s=0$. In one case, $v$ is the medium function; in the other, it is the velocity. Geometrical optics deals with rays and associated wavesurfaces. The same ray-wave relationship must, therefore, also be available for Newtonian mechanics. Starting with this important observation, Prof. J. L. Synge builds up the theory of "relativistic geometrical mechanics", in analogy with Hamilton's geometrical optics. Sources of light are replaced by space-time events, radiating world lines into the future; the wave-surfaces are three-dimensional surfaces in space-time-in fact, de Broglie waves, moving with a velocity which can be calculated from that of the associated ray.

If the concepts of wave-length, secondary waves, etc., are added semi-intuitively to the structure of geometrical optics, the resulting scheme (physical optics) suffices to give an adequate description of most optical phenomena. It provides easy solutions of problems where Maxwell's theory of electromagnetic waves would be difficult to apply. By analogy, Prof. Synge argues that a "primitive quantisation" of the scheme of relativistic geometrical mechanics (introduced by postulating that adjacent three-waves of equal phase are separated by a quantum of action $h$ ) would stand in the same relation to Schrödinger's wave mechanics as physical optics does to Maxwell's theory. A number of special cases are then discussed, among them the case of a particle in an electromagnetic field, and the case of the hydrogen atom.

Although one may wonder if Prof. Synge has not exhausted the possibilities of obtaining exact solutions, the novelty of his point of view is attractive and refreshing. The difficulty of the monograph comes from the relative unfamiliarity of the optical method of Hamilton, which had to be used, and the rather unfamiliar terminology. However, the effort of learning the new point of view is indeed very rewarding.

Abdus Salam

Three Abietaceous Cones from the Wealden of Belgium

(Mémoire No. 125 de l'Institut Royal des Sciences Naturelles de Belgique.) Par K. L. Alvin. Pp. $42+$ 5 plates. (Bruxelles: Institut Royal des Sciences Naturelles de Belgique, 1953.)

T this sumptuous monograph, a beginning is made 1 to the detailed description and classification of some of the remarkably well-preserved fossil plants in the collections of the late Prof. C. Bornmer, from Wealden elays in the Hainaut district of Belgium. 'The plants are preserved as a kind of lignite, in clay, and they have yielded to examination by microtome sections prepared by softening the material and then embedding in celloidin. In the cones here described, it has thus been possible to make a critical study of anatomical features, including the vascularization of the seed scales.

The three species of cones identified illustrate the importance and diversification attained by tho family of the Abietacese at this critical horizon in early Cretaceous times. The author reserves judgment on generic affinity and uses the form genus
Pityostrobus Nathorst for all of them, having regard to the absence of information on associated vegetative characters. It has been possible, however, to define the species on a range of characters which is unusually wide for fossilized conifers. $P$. andraei (Coemans), a widely-spread species, and P. bommeri Alvin probably both represent extinct species of the genus $P$ inus ; while $P$. corneti (Coemans), which is taxonomically perhaps the most interesting of the three, shares some characters with Cedrus and some with Keteleeria, and no doubt represents an extinct genus.

The continuation of such detailed studies of Cretaceous and of younger members of the Abietaceae should do much to elucidate the later evolution of this highly successful family, and thus add to the remarkable history of the Coniferales which has been placed on so well documented a basis by the work of Florin on the earlier members.

\section{Organic Syntheses}

An Annual Publication of Satisfactory Methods for the Preparation of Organic Chemicals. Edited by Charles C. Price. Vol. 33, 1953. Pp. vi+115. (New York: John Wiley and Sons, Inc.; London : Chapman and Hall, Ltd., 1953.) 28s, net.

THIS issue maintains the reputation of the series by recording reliable practical details for a further selection of forty compounds, of varied nature and interest. Among them, creosol (2-methoxy$p$-cresol) is obtained by treating an ethanolic solution of vanillin with amalgamated zine and concentrated hydrochloric acid, the aldehyde group being reduced to methyl; dimethylketene distils over in 50 per cent yield as a solution in ethyl acetate when $\alpha$-bromoisobutyryl bromide is added dropwise to boiling ethyl acetate and zinc turnings in an atmosphere of pure dry nitrogen under a pressure of $300 \mathrm{~mm}$. mercury; and 9-fluorenecarboxylic acid results almost quantitatively when benzilic acid is stirred with pure benzene and anhydrous aluminium chloride. Other preparations include nicotinonitrile from nicotinamide; $\alpha$-phenylcinnamic acid, and pure cis-stilbene therofrom; pyridine- $\mathrm{N}$-oxide from pyridine and 40 per cent peracetic acid; and stearone from stearic acid. The cumulative index covers Vols. $30-33$ of the series. An inserted leaflet directs attention to the explosive nature of the sodium salt of nitromalonaldehyde, mentioned in Vol. 32.

JoHN RraD

The Year's Work in Librarianship

Volume 17, 1950. Edited for the Library Association by W. A. Munford. Pp. $x+270$. (London: Library Association, 1954.) 40s.

THIS is the last of a series of seventeen volumes, for it is considered that Library Science Abstracts, together with the projected "Five Years' Work in Librarianship", the first volume of which will cover the period 1951-55, will better meet the needs of librarians. Besides the usual book list, this final volume includes chapters on national and university libraries by A. C. Townsend, special libraries (B. C. Vickery), library co-operation (C. C. Adams), library buildings (E. F. Patterson), library practice (G. R. Davies, M. Piggott and W. C. Berwick Sayers), and subject bibliography (P. D. Record). As usual, the volume is well produced, and though the claim is justified that present needs will be better met in other ways, some will nevertheless regret the displacement of a convenient reference work which has proved its value. 